\title{
Status of Body Mass Index(BMI) \& Waist Circumference (WC) in Patients of Chronic Obstructive Pulmonary Disease(COPD) \& Relationship With Severity.
}

\author{
Bhardwaj G, Jain V K, Mishra M, Nayyar P, Sharma A \\ Department Of Respiratory Medicine, Mahatma Gandhi Medical College And Hospital, Jaipur, Rajasthan
}

\begin{abstract}
Aims \& Objective - To study the BMI \& Waist circumference in patients of COPD \& their correlation with severity of disease.

Material \& method-This prospective study was conducted in Dept. of Respiratory Medicine MGMC\&H, Jaipur from Jan 2015 to March 2016. Patients were diagnosed COPD according to GOLD update(2015) \& staging(severity) was done according to Spirometry criteria. Waist circumference was measured \& BMI was calculated (WHO criteria) in all cases. BMI \& Waist circumference were analyzed \& co-related with severity of disease.

Results-120(82M, 38F) patients of COPD was analyzed .Out of 120 patients stage I were 8(6.67\%),II 41(34.17), III 38(31.67\%) \& stage IV 33(27.50). BMI in stage 1,2,3,4 were 22.9, 24.86, 21.38, 18.36 respectively with mean BMI 21.84kg/m2. Waist circumference in stage 1,2,3,4 were 79.63, 91.34,85.13,76.06 respectively. BMI and waist circumference decreases with increasing severity of COPD which was statistically significant

Conclusion -BMI \& waist circumference decreases more in severe \& very severe disease. Follow up monitoring of BMI with nutritional status is required for better management of COPD patients.
\end{abstract}

Keywords: Body Mass Index, Waist circumference, COPD

\section{Introduction}

Chronic obstructive pulmonary disease (COPD) is a significant cause of worldwide morbidity and mortality and considered to be systemic disease with widespread extra-pulmonary manifestation ${ }^{(1)}$.It is associated with muscles atrophy \& decreased strength leading to low fat free mass causing low BMI with severity of disease leading to increase exercise intolerance, morbidity and mortality. Waist circumference is also important anthroprometric profile which should be measured in COPD patients. Therefore we plan to study status of BMI $\&$ Waist circumference in patients of COPD \& correlate both with its severity.

\section{Material And Methods}

120 patients of COPD reporting to the Dept. of Respiratory Medicine from Jan 2015 to March 2016 at Mahatma Gandhi Hospital, Jaipur. COPD diagnosis \& grading of severity was done as per GOLD guidelines $2015^{(2)}$. BMI was calculated by Wt $(\mathrm{kg}) / \mathrm{ht}^{2}\left(\mathrm{~m}^{2}\right)$ \& classified according to WHO criteria ${ }^{(3)}$. Waist Circumference (WC) was measured at approximate midpoint between lower margin of last palpable rib \& top of ilaic crest according to WHO STEPS protocol. Correlation of BMI \& WC with severity of COPD was assessed.

\section{Results}

Out of 120 patients $82(68.3 \%)$ males \& $38(31.67 \%)$ were females, with male to female ratio $2.1: 1$. Mean age of studied patients was $59.79 \pm 8.46$ years, ranging from $40-83$ years. Mean age of males and females was $61.085 \pm 8.56$ and $57 \pm 7.644$ respectively.

Table 1: Gender Profile of COPD Cases

\begin{tabular}{|l|l|l|}
\hline Gender & No. & \% \\
\hline Male & 82 & 68.3 \\
\hline Female & 38 & 31.7 \\
\hline Total & 120 & 100 \\
\hline
\end{tabular}


Table 2: Age Profile of COPD Cases

\begin{tabular}{|l|l|l|l|l|l|l|}
\hline \multirow{2}{*}{ Age Group } & \multicolumn{2}{|l|}{$\begin{array}{l}\text { Female } \\
(\mathbf{n = 3 8 )}\end{array}$} & \multicolumn{2}{l|}{$\begin{array}{l}\text { Male } \\
\text { (n=82) }\end{array}$} & \multicolumn{2}{l|}{$\begin{array}{l}\text { Total } \\
\text { (n=120) }\end{array}$} \\
\cline { 2 - 7 } & No & $\%$ & No & $\%$ & No & $\%$ \\
\hline$<50$ & 7 & 18.42 & 10 & 12.20 & 17 & 14.17 \\
\hline 51 to 60 & 21 & 55.26 & 36 & 43.90 & 57 & 47.50 \\
\hline 61 to 70 & 8 & 21.05 & 25 & 30.49 & 33 & 27.50 \\
\hline$>70$ & 2 & 5.26 & 11 & 13.41 & 13 & 10.83 \\
\hline Total & 38 & 100.00 & 82 & 100.00 & 120 & 100.00 \\
\hline
\end{tabular}

Chi-square $=3.849$ with 3 degrees of freedom; $\mathrm{P}=0.374$

Mean weight was $56.24 \pm 14.21 \mathrm{~kg}$. Mean BMI was $21.84 \pm 5.36 \mathrm{~kg} / \mathrm{m}^{2}$.Mean waist circumference was $92.63 \pm 8.1 \mathrm{~cm}$.

Table 3: Anthropometric Profile in COPD Cases

\begin{tabular}{|l|l|l|l|l|l|l|}
\hline \multirow{2}{*}{ BMI } & \multicolumn{2}{l|}{$\begin{array}{l}\text { Female } \\
(\mathbf{n = 3 8})\end{array}$} & \multicolumn{2}{l|}{$\begin{array}{l}\text { Male } \\
(\mathbf{n = 8 2})\end{array}$} & \multicolumn{2}{l|}{$\begin{array}{l}\text { Total } \\
(\mathbf{n = 1 2 0})\end{array}$} \\
\cline { 2 - 8 } & No & \% & No & $\%$ & No & $\%$ \\
\hline$<18.5$ (Underweight) & 13 & 34.21 & 24 & 29.268 & 37 & 30.83 \\
\hline 18.5 to24.9 (Normal) & 10 & 26.32 & 43 & 52.439 & 53 & 44.17 \\
\hline 25 to 30 (Overweight) & 12 & 31.58 & 11 & 13.415 & 23 & 19.17 \\
\hline$>30$ (Obese) & 3 & 7.89 & 4 & 4.878 & 7 & 5.83 \\
\hline Total & 38 & 100 & 82 & 100 & 120 & 100 \\
\hline
\end{tabular}

Chi-square $=9.093$ with 3 degrees of freedom; $\mathrm{P}=0.036 \mathrm{~S}$

Among COPD cases, majority of patients 53(44.17\%) had normal BMI, followed by underweight $37(30.83 \%)$, overweight $23(19.17 \%)$ and obese $(5.83 \%)$.Numbers of females were significantly more than males in overweight patients (p-0.036).

Table 4: Staging of COPD as per Spirometry

\begin{tabular}{|l|l|l|}
\hline Stage & No. & $\%$ \\
\hline 1 & 8 & 6.67 \\
\hline 2 & 41 & 34.17 \\
\hline 3 & 38 & 31.67 \\
\hline 4 & 33 & 27.50 \\
\hline Total & 120 & 100 \\
\hline
\end{tabular}

Maximum number of patients $41(34.17 \%)$ were in stage $2,38(31.67 \%)$ in stage 3, $33(27.5 \%)$ in stage 4 and minimum $8(6.67 \%)$ in stage 1 .

Table 5: Co-relation of BMI,WC with severity of COPD

\begin{tabular}{|l|l|l|l|l|l|}
\hline COPD & Stage 1 & Stage 2 & Stage 3 & Stage 4 & \\
\hline BMI & $22.9 \pm 2.04$ & $24.86 \pm 5.2$ & $21.38 \pm 5.2$ & $18.36 \pm 4.03$ & $<0.001$ \\
\hline WC & $79.63 \pm 13.11$ & $91.34 \pm 14.42$ & $85.13 \pm 14.67$ & $76.06 \pm 13.46$ & $<0.001$ \\
\hline
\end{tabular}

BMI in stage 1,2,3,4 were 22.9, 24.86, 21.38, 18.36 respectively with mean BMI $21.84 \mathrm{~kg} / \mathrm{m} 2$. Waist circumference in stage 1,2,3,4 were 79.63 , 91.34,85.13,76.06 respectively. BMI and waist circumference decreases with increasing severity of COPD which was statistically significant.

\section{Discussion}

Despite adequate calorie intake cachexia is common co-morbidity of COPD patients due to multifactorial mechanisms. Low BMI is considered poor prognostic marker of COPD. Our study comprises of 
120 COPD cases (68.3\% males and $31.67 \%$ females) with male to female ratio $2.1: 1$ which is similar to Indian council of medical research (ICMR) study $(\mathrm{M}: \mathrm{F} ; 1.56: 1)^{(4)}$.

In present study patients were in age of $40-83$ years with mean age of $59.8 \pm 8.46$ years. $3 / 4^{\text {th }}$ of patients lie in age groups of 51-70 years both in males $(67.8 \%)$ and females $(32.2 \%)$. There was no significant difference in mean age of either sex. Dhadke et $\mathrm{l}^{(5)}$ also reported similar results.

As per spirometry assessment in our study almost $1 / 3^{\text {rd }}$ patients were in each stage 2 and 3 , followed by stage 4 and minimum in stage $1(6.7 \%)$. Results were variable in other studies while maximum cases were reported in moderate to severe COPD. In our study, BMI/ WC decreases with progression of COPD severity Similar to a study done by Sajal de et al ${ }^{(8)}$, thereby indicating under weight is more in severe COPD.

In comparison to our \& other Indian studies, western COPD population have lower prevalence of underweight as majority of patients in India report in late stages of COPD where mean BMI decreases which is also supported by Sajal De et al. In our study we observed that mean BMI, waist circumference decreases significantly with increasing severity of COPD which was statistically significant Increased BMI, waist circumference in early stages of COPD, makes them more prone for metabolic syndrome.

\section{Conclusion}

Obesity in COPD patients act as long term killer with short term protection than the rapid effect of cachexia-malnutrition leading to high morbidity \& mortality. BMI,WC reduces with the progression of COPD. So both together affect its outcome. Thus follow up monitoring of BMI \& nutritional status is essential for better outcome.

\section{References}

[1]. Jindal S.K. COPD: The unrecognized epidemic in India, JAPI, Feb 2012;60:14-16

[2]. COPDGoldGuidelineUpdate2015,www.goldCOPD.org/uploads/users/files/GOLD_report

[3]. "BMI Classification”. Global Database on Body Mass Index. World Health Organization. 2006

[4]. Jindal SK. Emergence of COPD as an epidemic in India. Indian J Med Res. 2006 Dec;124:619-30.

[5]. Dhadke VN, Dhadke SV, Raut N. Clinical Profile in chronic obstructive lung disease patients and their evaluation with spirometry and2D Echo.International J of Current Research. 2015Feb;7(02):12480-8.

[6]. Akpinar EE, Akpinar S, Ertek S, Sayin E, Gulhan M. Systemic inflammation and metabolic syndrome in stable COPD patients. Tuberk Toraks. 2012; 60(3):230-7.

[7]. Ozgen AA, Konyar AI, Serter S, Sakar CA, Celik P, Taneli F, et al. Metabolic syndrome and carotid intimamedia thickness in chronic obstructive pulmonary disease. Multidiscip Respir Med. 2013;8(1):61.

[8]. Sajal De. Body Mass Index Among Patients With Chronic Obstructive Pulmonary Disease. Indian J Physiol Pharmacol. 2012;56(4):353-8 